Research Article

\title{
Sombor energy and Hückel rule
}

\author{
Ivan Gutman*, Izudin Redžepović \\ Faculty of Science, University of Kragujevac, 34000 Kragujevac, Serbia
}

(Received: 28 January 2022. Accepted: 18 February 2022. Published online: 21 February 2022.)

(c) 2022 the authors. This is an open access article under the CC BY (International 4.0) license (www.creativecommons.org/licenses/by/4.0/).

\begin{abstract}
The Sombor index, a recently invented vertex-degree-based graph invariant, is insensitive to the size of cycles contained in a graph. In contrast to this, the Sombor energy, the sum of absolute values of the Sombor matrix, is found to have a significant cycle-size dependence. In the case of bipartite graphs, this dependence is analogous to the Hückel ( $4 n+2)-r u l e:$ cycles of size $4,8,12, \ldots$ decrease, and cycles of size $6,10,12, \ldots$ increase the Sombor energy. A theorem corroborating this empirical observation is offered.
\end{abstract}

Keywords: energy (of a graph); Sombor index; Sombor energy; graph energy; topological index; degree (of a vertex).

2020 Mathematics Subject Classification: 05C07, 05C09, 05C50.

\section{Introduction}

Since the introduction of the concept of Sombor index [10], its chemical applications were extensively studied [1,2,6,24, $28,29]$. However, one chemically important property of the Sombor index, namely its dependence on the size of the cycles contained in a (molecular) graph, was never examined. The reason for this is evident: It is easy to recognize that neither the Sombor index nor any of the numerous other vertex-degree-based topological indices have any noteworthy cycle-size dependence. The examples depicted in Figure 1 may suffice to corroborate this conclusion.

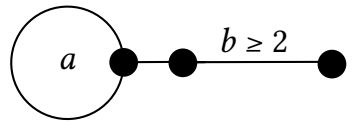

$X_{a, b}$

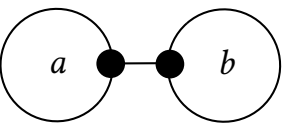

$Y_{a, b}$

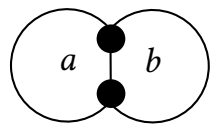

$Z_{a, b}$

Figure 1: Examples of molecular graphs whose Sombor index is independent of the size $(a, b)$ of their cycles; $n=$ number of vertices; $S O\left(X_{a, b}\right)=\sqrt{5}+3 \sqrt{13}+(n-4) \sqrt{8} ; S O\left(Y_{a, b}\right)=\sqrt{18}+4 \sqrt{13}+(n-4) \sqrt{8} ; S O\left(Z_{a, b}\right)=\sqrt{18}+4 \sqrt{13}+(n-4) \sqrt{8}$.

Recently, a detailed theory of degree-based matrices and the respective degree-based energies was elaborated [4,11,13, $17,23,32]$. Within this theory, also the concept of Sombor matrix and Sombor energy was introduced [7,12,15,19,34]. In this paper, we show that, in contrast to the Sombor index, the Sombor energy possesses a pronounced cycle-size dependence. In particular, we establish the existence of a Hückel-rule-type regularity for the Sombor energy.

Recall that the famous Hückel $(4 n+2)$-rule claims that cycles of size $6,10,14, \ldots$ stabilize, whereas cycles of size 4 , $8,12, \ldots$ destabilize a cyclic conjugated $\pi$-electron system $[8,18,20,21,27]$. In terms of graph energy $[5,14,22]$ or other eigenvalue-based topological indices [30], cycles of size $4,8,12, \ldots$ have a positive (increasing) effect on graph energy, whereas the effect of $4,8,12, \ldots$ cycles is negative. In what follows, we demonstrate that an analogous regularity holds for the Sombor energy. A characteristic example is shown in Figure 2.

\section{Mathematical introduction}

Let $G$ be a simple graph with vertex set $\mathbf{V}(G)$ and edge set $\mathbf{E}(G)$. Because we are interested in effects caused by even cycles, it will be assumed that $G$ is connected and bipartite and, of course, contains at least one cycle. Recall that in bipartite graphs, the size of all cycles is even.

If the vertices $u, v \in \mathbf{V}(G)$ are adjacent, then the edge connecting them is denoted by $u v$. The number of edges incident to a vertex $v$ is the degree of vertex $v$, and is denoted by $d_{v}$. 


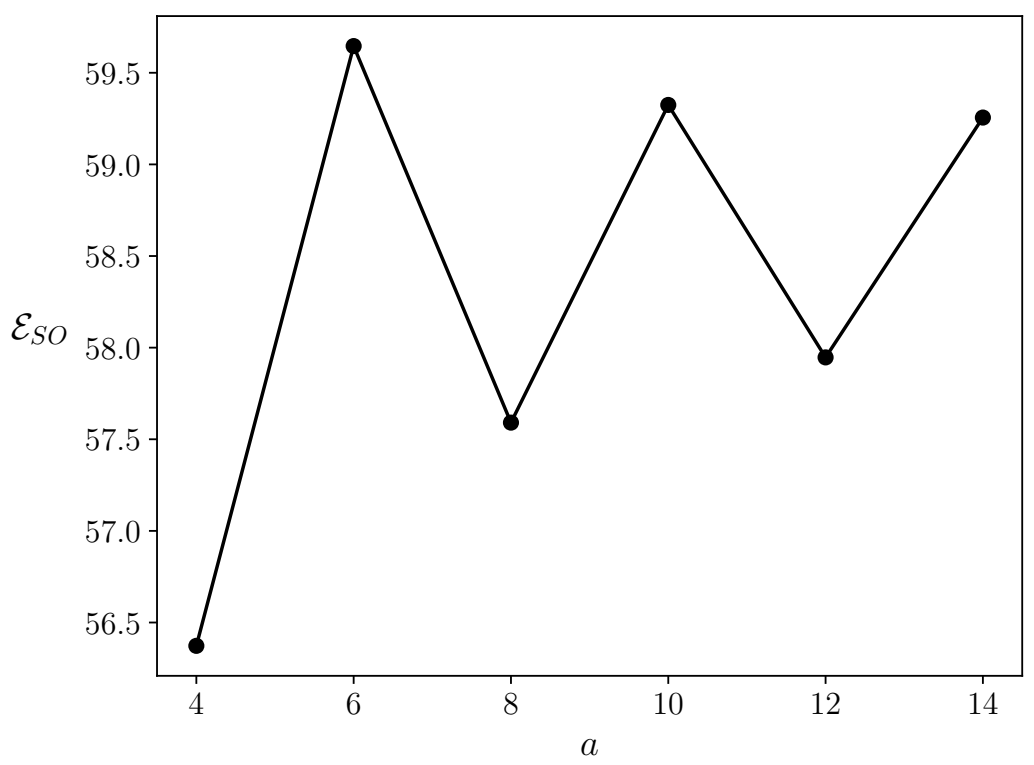

Figure 2: The Sombor energy of the unicyclic graph $X_{a, b}, a+b=16$, plotted versus the size $a$ of its cycle.

The Sombor index is defined as [10]

$$
S O=S O(G)=\sum_{u v \in \mathbf{E}(G)} \sqrt{d_{u}^{2}+d_{v}^{2}} .
$$

Bearing this in mind, if $\mathbf{V}(G)=\left\{v_{1}, v_{2}, \ldots, v_{n}\right\}$ is the vertex set of the graph $G$, then its Sombor matrix $\mathbf{A}_{S O}(G)=\left(a_{S O}\right)_{i j}$ is the symmetric matrix of order $n$, whose elements are

$$
\left(a_{S O}\right)_{i j}=\left\{\begin{array}{cl}
\sqrt{d_{v_{i}}^{2}+d_{v_{j}}^{2}} & \text { if } v_{i} v_{j} \in \mathbf{E}(G) \\
0 & \text { if } v_{i} v_{j} \notin \mathbf{E}(G) \\
0 & \text { if } i=j .
\end{array}\right.
$$

If the eigenvalues of $\mathbf{A}_{S O}(G)$ are $\lambda_{1}, \lambda_{2}, \ldots, \lambda_{n}$, then the Sombor energy of the graph $G$ is defined as

$$
\mathcal{E}_{S O}=\mathcal{E}_{S O}(G)=\sum_{i=1}^{n}\left|\lambda_{i}\right|
$$

For the hitherto established properties of the Sombor energy see [7,12,15, 19,34].

\section{Numerical work}

In order to get an idea on the cycle-size dependence of the Sombor energy, we calculated the $\mathcal{E}_{S O}$-values of several classes of molecular graphs, in which the size of the cycles varies. In Tables 1 and 2 are presented such results for the graphs $X$, $Y$, and $Z$, depicted in Figure 1.

Table 1: Sombor energies of the unicyclic graphs $X_{a, b}$ whose structure is found in Figure 1. The Hückel-rule-type dependence on cycle size is clearly visible.

\begin{tabular}{|c|c|c||c|c|c|}
\hline $\begin{array}{c}\text { number of } \\
\text { vertices }\end{array}$ & $\begin{array}{c}\text { size of } \\
\text { cycle }\end{array}$ & $\mathcal{E}_{S O}(X)$ & $\begin{array}{c}\text { number of } \\
\text { vertices }\end{array}$ & $\begin{array}{c}\text { size of } \\
\text { cycle }\end{array}$ & $\mathcal{E}_{S O}(X)$ \\
\hline 8 & 4 & 27.392 & 12 & 8 & 43.132 \\
8 & 6 & 30.984 & 12 & 10 & 45.006 \\
10 & 4 & 34.662 & 14 & 4 & 49.145 \\
10 & 6 & 38.121 & 14 & 6 & 52.462 \\
10 & 8 & 35.895 & 14 & 8 & 50.365 \\
12 & 4 & 41.910 & 14 & 10 & 52.154 \\
12 & 6 & 45.285 & 14 & 12 & 50.737 \\
\hline
\end{tabular}


Table 2: Sombor energies of bicyclic graphs $Y_{a, b}$ and $Z_{a, b}$ whose structure is found in Figure 1. A Hückel-rule-type dependence can be envisaged, but is somewhat less pronounced than in the case of $X_{a, b}$.

\begin{tabular}{|c|c|c||c|c|c|}
\hline $\begin{array}{c}\text { number of } \\
\text { vertices }\end{array}$ & $\begin{array}{c}\text { sizes of } \\
\text { cycles }\end{array}$ & $\mathcal{E}_{S O}(Y)$ & $\begin{array}{c}\text { number of } \\
\text { vertices }\end{array}$ & $\begin{array}{c}\text { sizes of } \\
\text { cycles }\end{array}$ & $\mathcal{E}_{S O}(Z)$ \\
\hline 8 & 4,4 & 30.658 & 8 & 4,6 & 33.914 \\
10 & 4,6 & 40.038 & 10 & 4,8 & 40.986 \\
12 & 4,8 & 46.162 & 10 & 6,6 & 43.638 \\
12 & 6,6 & 51.284 & 12 & 4,10 & 48.165 \\
14 & 4,10 & 54.172 & 12 & 6,8 & 49.260 \\
14 & 6,8 & 56.018 & 14 & 4,12 & 55.394 \\
& & & 14 & 6,10 & 57.670 \\
& & & 14 & 8,8 & 56.028 \\
\hline
\end{tabular}

\section{A theorem on cycle dependence of Sombor energy}

The Sombor matrix $\mathbf{A}_{S O}(G)$ can be viewed as the adjacency matrix of an ordinary graph with appropriately weighted edges. Let, thus, $G$ be a bipartite graph, such that its edge $e=u v$ has a weight $w(e)=w(u v)$. Recall that in our case, $w(u v)=\sqrt{d_{u}^{2}+d_{v}^{2}}>0$.

Since $G$ is bipartite, its characteristic polynomial of $G$ has the form $[3,19]$

$$
\phi(G, \lambda)=\sum_{k \geq 0}(-1)^{k} b(G, k) \lambda^{n-2 k}
$$

so that all its coefficients $b(G, k)$ are non-negative. According to the Sachs theorem [3,19,25,31]

$$
(-1)^{k} b(G, k)=\sum_{\sigma \in \mathcal{S}_{2 k}(G)}(-1)^{p(\sigma)} 2^{c(\sigma)} w(\sigma)
$$

where $\mathcal{S}_{k}(G)$ is the set of all Sachs graphs of $G$ possessing exactly $2 k$ vertices, and where $\sigma$ is an element of $\mathcal{S}_{2 k}(G)$, containing $p(\sigma)$ components, of which $c(\sigma)$ are cycles. The weight $w(\sigma)$ of the Sachs graph $\sigma$ is equal to the product of the weights of its components. If the isolated edge $u v$ (consisting of two vertices) is a component of $\sigma$, then its weight is $w_{u v}^{2}$. If a cycle $Z$ is a component of $\sigma$, then its weight is the product of weights of its edges [21,31].

The energy of the weighted bipartite graph $G$, i.e., the sum of the absolute values of its eigenvalues, is related with the coefficients of the characteristic polynomial by means of the Coulson integral formula [9,22]

$$
\mathcal{E}(G)=\frac{2}{\pi} \int_{0}^{\infty} \frac{d x}{x^{2}} \ln \sum_{k \geq 0} b(G, k) x^{2 k}
$$

which means that the energy is a monotonically increasing function of each of the coefficients.

Let $Z$ be a cycle of the graph $G$, and let its size be $h$, an even number. Label the edges of $Z$ consecutively by $e_{1}, e_{2}, \ldots, e_{h}$. We are interested in the effect of the cycle $Z$ on the energy of the graph $G$. In view of the Sachs and Coulson formulas (1) and (2), this effect will manifest itself via the Sachs graphs which contain $Z$.

Let $\sigma_{1} \in \mathcal{S}_{2 k}(G)$ be a Sachs graph containing the cycle $Z$. Then the components of $\sigma_{1}$, other than $Z$ form a Sachs graph $\sigma^{*} \in \mathcal{S}_{2 k-h}(G-Z)$. It holds,

$$
\begin{aligned}
p\left(\sigma_{1}\right) & =1+p\left(\sigma^{*}\right) \\
c\left(\sigma_{1}\right) & =1+c\left(\sigma^{*}\right) \\
w\left(\sigma_{1}\right) & =w(Z) \cdot w\left(\sigma^{*}\right)
\end{aligned}
$$

which by Equation (1) yields

$$
\sum_{\sigma_{1}}(-1)^{p(\sigma)} 2^{c(\sigma)} w(\sigma)=-2 w(Z) \sum_{\sigma^{*}}(-1)^{p(\sigma)} 2^{c(\sigma)} w(\sigma)=-2 w(Z)(-1)^{k-h / 2} b(G-Z, k-h / 2) .
$$

If there is a Sachs graph $\sigma_{1}$, then there must exist another Sachs graph $\sigma_{2}$ which instead of the cycle $Z$ contains the edges $e_{1}, e_{3}, \ldots, e_{h-1}$. There also must exist a Sachs graph $\sigma_{3}$ containing the edges $e_{2}, e_{4}, \ldots, e_{h}$. For them,

$$
p\left(\sigma_{2}\right)=h / 2+p\left(\sigma^{*}\right)
$$




$$
\begin{aligned}
c\left(\sigma_{2}\right) & =c\left(\sigma^{*}\right) \\
w\left(\sigma_{2}\right) & =w\left(e_{1}\right)^{2} w\left(e_{3}\right)^{2} \cdots w\left(e_{h-1}\right)^{2} \cdot w\left(\sigma^{*}\right) \\
p\left(\sigma_{3}\right) & =h / 2+p\left(\sigma^{*}\right) \\
c\left(\sigma_{3}\right) & =c\left(\sigma^{*}\right) \\
w\left(\sigma_{3}\right) & =w\left(e_{2}\right)^{2} w\left(e_{4}\right)^{2} \cdots w\left(e_{h}\right)^{2} \cdot w\left(\sigma^{*}\right)
\end{aligned}
$$

and, in an analogous manner as above

$$
\sum_{\sigma_{2}}(-1)^{p(\sigma)} 2^{c(\sigma)} w(\sigma)=(-1)^{h / 2} w\left(e_{1}\right)^{2} w\left(e_{3}\right)^{2} \cdots w\left(e_{h-1}\right)^{2}(-1)^{k-h / 2} b(G-Z, k-h / 2)
$$

and

$$
\sum_{\sigma_{3}}(-1)^{p(\sigma)} 2^{c(\sigma)} w(\sigma)=(-1)^{h / 2} w\left(e_{2}\right)^{2} w\left(e_{4}\right)^{2} \cdots w\left(e_{h}\right)^{2}(-1)^{k-h / 2} b(G-Z, k-h / 2) .
$$

The total effect of the cycle $Z$ on the coefficient $b(G, k)$ is thus

$$
e f(G, Z)=(-1)^{k} \sum_{\sigma_{1}, \sigma_{2}, \sigma_{3}}(-1)^{p(\sigma)} 2^{c(\sigma)} w(\sigma)
$$

which by taking into account Equations (3)-(5) yields

$$
e f(G, Z)=\left[w\left(e_{1}\right)^{2} w\left(e_{3}\right)^{2} \cdots w\left(e_{h-1}\right)^{2}+w\left(e_{2}\right)^{2} w\left(e_{4}\right)^{2} \cdots w\left(e_{h}\right)^{2}-2(-1)^{h / 2} w(Z)\right] b(G-Z, k-h / 2) .
$$

Bearing in mind that $w(Z)=w\left(e_{1}\right) w\left(e_{2}\right) \cdots w\left(e_{h-1}\right) w\left(e_{h}\right)$,

$$
\begin{aligned}
e f(G, Z) & =\left[\left(w\left(e_{1}\right) w\left(e_{3}\right) \cdots w\left(e_{h-1}\right)\right)^{2}+\left(w\left(e_{2}\right) w\left(e_{4}\right) \cdots w\left(e_{h}\right)\right)^{2} \pm 2 w\left(e_{1}\right) w\left(e_{2}\right) \cdots w\left(e_{h}\right)\right] b(G-Z, k-h / 2) \\
& =\left[w\left(e_{1}\right) w\left(e_{3}\right) \cdots w\left(e_{h-1}\right) \pm w\left(e_{2}\right) w\left(e_{4}\right) \cdots w\left(e_{h}\right)\right]^{2} b(G-Z, k-h / 2) .
\end{aligned}
$$

We now arrive at our main result:

Theorem 4.1. (a) If the size $h$ of the cycle $Z$ contained in the bipartite graph $G$ is divisible by 4 , i.e., if $h / 2$ is even, then the respective energy effect is

$$
e f(G, Z)=\left[w\left(e_{1}\right) w\left(e_{3}\right) \cdots w\left(e_{h-1}\right)-w\left(e_{2}\right) w\left(e_{4}\right) \cdots w\left(e_{h}\right)\right]^{2} b(G-Z, k-h / 2) .
$$

(b) If the size $h$ of the cycle $Z$ contained in the bipartite graph $G$ is not divisible by 4 , i.e., if $h / 2$ is odd, then the respective energy effect is

$$
e f(G, Z)=\left[w\left(e_{1}\right) w\left(e_{3}\right) \cdots w\left(e_{h-1}\right)+w\left(e_{2}\right) w\left(e_{4}\right) \cdots w\left(e_{h}\right)\right]^{2} b(G-Z, k-h / 2) .
$$

By Theorem 4.1, ef $(G, Z) \geq 0$ for all edge-weighted bipartite graphs $G$ and all their cycles $Z$. This means that all cycles increase the value of the coefficients $b(G, k)$ and thus, by Equation (2), increase the value of $\mathcal{E}(G)$. However, in the case of cycles of size $4,8,12, \ldots$, this increasing effect is smaller than for the cycles of size $6,10,14, \ldots$, and may be equal to zero. Needless to say that these conclusions apply also to the Sombor energy $\mathcal{E}_{S O}$.

One should note that the validity of both Theorem 4.1 and its proof are restricted to bipartite graphs. Their extension to even cycles in non-bipartite graphs seems to be not easy and remains a task for the future.

Since the term $b(G-Z, k-h / 2)$ is non-negative, and since (in our case) all edge-weights are positive-valued, Theorem 4.1 is in good agreement with the empirically observed fact that the Sombor energy has a Hückel-rule-type cycle-dependence. Yet, it should not be considered as a proper proof of this Hückel-rule-type cycle-dependence.

\section{A historical remark}

This paper is dedicated to Professor Nenad Trinajstić. Therefore, it should be noted that studies related to the Hückel rule were the topics of Trinajstić's earliest researches, published exactly half a century ago [16,26]. Also later, he remained interested in problems of this kind (e.g., [8,20,33]). The paper [16] happens to be the first scientific publication of one of the present authors (I.G.), who then was one of Professor Trinajstić's first students. 


\section{Acknowledgment}

Izudin Redžepović was supported by the Serbian Ministry of Education, Science and Technological Development (Grant No. 451-03-9/2021-14/200122).

\section{References}

[1] S. Alikhani, N. Ghanbari, Sombor index of polymers, MATCH Commun. Math. Comput. Chem. 86 (2021) 715-728.

[2] R. Cruz, I. Gutman, J. Rada, Sombor index of chemical graphs, Appl. Math. Comput. 399 (2021) \#126018.

[3] D. Cvetković, P. Rowlinson, S. Simić, An Introduction to the Theory of Graph Spectra, Cambridge Univ. Press, Cambridge, 2010.

[4] K. C. Das, I. Gutman, I. Milovanović, E. Milovanović, B. Furtula, Degree-based energies of graphs. Linear Algebra Appl. 554 (2018) 185-204.

[5] E. Estrada, M. Benzi, What is the meaning of the graph energy after all?, Discrete Appl. Math. 230 (2017) 71-77.

[6] X. Fang, L. You, H. Liu, The expected values of Sombor indices in random hexagonal chains, phenylene chains and Sombor indices of some chemical graphs, Int. J. Quantum Chem. 121 (2021) \# 26740.

[7] K. J. Gowtham, N. N. Swamy, On Sombor energy of graphs, Nanosystems: Phys. Chem. Math. 12 (2021) 411-417.

[8] A. Graovac, I. Gutman, N. Trinajstić, Topological Approach to the Chemistry of Conjugated Molecules, Springer, Berlin, 1977.

[9] I. Gutman, Acyclic systems with extremal Hückel $\pi$-electron energy, Theor. Chim. Acta 45 (1977) 79-87.

[10] I. Gutman, Geometric approach to degree-based topological indices: Sombor indices, MATCH Commun. Math. Comput. Chem. 86 (2021) 11-16.

[11] I. Gutman, Comparing degree-based energies of trees, Contrib. Math. 4 (2021) 1-5.

[12] I. Gutman, Spectrum and energy of the Sombor matrix, Mil. Tech. Cour. 69 (2021) 551-561.

[13] I. Gutman, Estimating vertex-degree-energies, Mil. Tech. Cour. $\mathbf{7 0}$ (2022) 13-23.

[14] I. Gutman, B. Furtula, The total $\pi$-electron energy saga, Croat. Chem. Acta 90 (2017) 359-368.

[15] I. Gutman, K. J. Gowtham, On characteristic polynomial and energy of Sombor matrix, Open J. Discrete Appl. Math. 4(3) (2021) 29-35.

[16] I. Gutman, M. Milun, N. Trinajstić, Hückel molecular orbital calculations of aromatic stabilization of annulenes, Croat. Chem. Acta 44 (1972) 207-213.

[17] I. Gutman, J. Monsalve, J. Rada, A relation between a vertex-degree-based topological index and its energy, Linear Algebra Appl. 636 (2022) $134-142$.

[18] I. Gutman, O. E. Polansky, Cyclic conjugation and the Hückel molecular orbital model, Theor. Chim. Acta 60 (1981) $203-226$.

[19] I. Gutman, I. Redžepović, J. Rada, Relating energy and Sombor energy, Contrib. Math. 4 (2021) 41-44.

[20] I. Gutman, N. Trinajstić, Graph theory and molecular orbitals. XV. The Hückel rule, J. Chem. Phys. 64 (1976) $4921-4925$.

[21] E. Hückel, Grundzüge der Theorie ungesättigter und aromatischer Verbindungen, Verlag Chemie, Berlin, 1940.

[22] X. Li, Y. Shi, I. Gutman, Graph Energy, Springer, New York, 2012.

[23] X. Li, Z. Wang, Trees with extremal spectral radius of weighted adjacency matrices among trees weighted by degree-based indices, Linear Algebra Appl. 620 (2021) 61-75.

[24] H. Liu, H. Chen, Q. Xiao, X. Fang, Z. Tang, More on Sombor indices of chemical graphs and their applications to the boiling point of benzenoid hydrocarbons, Int. J. Quantum Chem. 121 (2021) \#e26689.

[25] R. B. Mallion, N. Trinajstić, A. J. Schwenk, Graph theory in chemistry - Generalization of Sachs' formula, Z. Naturforsch. 29a (1974) 1481-1484.

[26] M. Milun, Ž. Sobotka, N. Trinajstić, Hückel molecular orbital calculations of the index of aromatic stabilization of polycyclic conjugated molecules, J. Org. Chem. 37 (1972) 139-141.

[27] M. Randić, Aromaticity of polycyclic conjugated hydrocarbons, Chem. Rev. 103 (2003) 3449-3606.

[28] Z. Raza, K. Naz, S. Ahmad, Expected values of molecular descriptors in random polyphenyl chains, Emerging Sci. J. 6 (2022) 151-165.

[29] I. Redžepović, Chemical applicability of Sombor indices, J. Serb. Chem. Soc. 86 (2021) 445-457.

[30] I. Redžepović, S. Radenković, B. Furtula, Effect of a ring onto values of eigenvalue-based molecular descriptors, Symmetry 13 (2021) \#1515.

[31] H. Sachs, Beziehungen zwischen den in einem Graphen enthaltenen Kreisen und seinem charakteristischen Polynom, Publ. Math. (Debrecen) 11 (1964) 119-134.

[32] Y. Shao, Y. Gao, W. Gao, X. Zhao, Degree-based energies of trees, Linear Algebra Appl. 621 (2021) 18-28.

[33] N. Trinajstić, Chemical Graph Theory, CRC Press, Boca Raton, 1983; 2nd Revised Edition, 1992.

[34] Z. Wang, Y. Mao, I. Gutman, J. Wu, Q. Ma. Spectral radius and energy of Sombor matrix of graphs, Filomat 35 (2021) $5093-5100$. 